\title{
Correction to: Consistent Condom Use by Female Sex Workers in Kolkata, India: Testing Theories of Economic Insecurity, Behavior Change, Life Course Vulnerability and Empowerment
}

\author{
Anne E. Fehrenbacher ${ }^{1} \cdot$ Debasish Chowdhury ${ }^{2} \cdot$ Toorjo Ghose $^{3}$. Dallas Swendeman ${ }^{4,5}$
}

Published online: 4 December 2017

(c) Springer Science+Business Media, LLC, part of Springer Nature 2017

\section{Correction to: AIDS Behav (2016) 20:2332-2345 https://doi.org/10.1007/s10461-016-1412-z}

The original version of this article unfortunately contained an error. The authors would like to correct the error with this erratum.

The binary variable for "Client offered more money for sex without a condom within the past five years" was miscoded in the opposite direction. This variable was not a significant determinant of any of the outcomes in the multivariate analysis: (1) used a condom with last occasional client; (2) used a condom with last regular client, or (3) used a condom with both client types. The multivariate results remain unchanged with this correction. However, the authors issue this erratum to correct the univariate descriptive statistics and interpretation of this variable in three parts of the article.

The original article can be found online at https://doi.org/10.1007/ s10461-016-1412-z.

Dallas Swendeman

dswendeman@mednet.ucla.edu

1 Department of Community Health Sciences, Fielding School of Public Health, University of California, Los Angeles, 650 Charles E Young Drive South, Los Angeles, CA 90095, USA

2 Public Health Foundation of India (PHFI), Sector-44, Plot No-47, Gurgaon 122003, India

3 School of Social Policy \& Practice, University of Pennsylvania, 3701 Locust Walk, Caster D17, Philadelphia 19104, USA

4 Center for HIV Identification, Prevention and Treatment Services (CHIPTS), Department of Psychiatry and Biobehavioral Science, David Geffen School of Medicine, University of California, Los Angeles, 10920 Wilshire Blvd., Suite 350, Los Angeles, CA 90024, USA

5 Department of Epidemiology, Fielding School of Public Health, 10920 Wilshire Blvd., Suite 350, Los Angeles 90024, USA
1. The proportion of respondents who reported being offered more money for sex without a condom by a client within the past five years was incorrectly stated as "a third" in the text of the results (p. 2337). The incorrect proportion should be replaced with "approximately two-thirds" in the last sentence of the paragraph on Condom Use Behaviors, on page 2337.

The correct sentence should read as:

"Although approximately two-thirds of respondents had been offered more money for sex without a condom within the past five years, only $18 \%$ reported accepting it."

2. The percentage and frequency of respondents who reported being offered more money for sex without a condom by a client within the past five years were incorrectly reported as " $31.5 \%$ " and " $\mathrm{n}=63$ ", respectively, in Table 1 (p. 2339). The incorrect values for this variable should be replaced with "68.5\%" and " $n=137$ ", respectively, in the Table 1 section on Sexual risk knowledge, attitudes, behaviors, on page 2339 .

The correct percentage and frequency for this variable in Table 1 are presented below.

3 . The proportion of respondents who reported being offered more money for sex without a condom by a client within the past 5 years was incorrectly explained in the text of the discussion (p. 2343). The following sentences should be replaced in the third paragraph, on page 2343:

"It should also be noted that the majority of FSW did not report being offered more money for sex without a condom,

Table 1 Demographic, financial, participation and empowerment characteristics of brothel-based female sex workers in Kolkata, India $(\mathrm{n}=200)$

\begin{tabular}{lllll}
\hline Variable & $\%$ & $\mathrm{n}$ & Mean & SD \\
\hline $\begin{array}{l}\text { Sexual risk knowledge, attitudes, behaviors } \\
\text { Client offered more money for sex with- } \\
\text { out condom past 5 years }\end{array}$ & 68.5 & 137 & & \\
\hline
\end{tabular}


which may suggest that the client population in this area does not have a strong preference for condomless sex, and/ or that clients are aware of both condom use norms and support for refusal by power brokers (i.e., madams) and peers. Durbar advocates with brothel power-brokers and clients to make condom use the status quo while mobilizing collective identity among sex workers through street rallies, conferences, and peer education campaigns to frame condom use as issues of workers' rights and occupational safety [51]."

The correct sentences should read as:

"Given that the majority of respondents reported that they had been offered more money for sex without a condom by a client within the past five years, it is notable that very few FSW reported accepting such an offer. The high rate of refusal to accept more money for condomless sex suggests that Durbar has been successful at framing consistent condom use as an issue of workers' rights and occupational safety. Durbar has established condom use norms by mobilizing collective identity among sex workers through street rallies, conferences, and peer education campaigns and by advocating with power brokers (i.e., madams) and peers to provide support for refusal [51]. Additional advocacy may be necessary to make condom use a normative preference among clients." 